\title{
FREE VIBRATIONS OF ROTATING ADHESIVELY BONDED ORTHOTROPIC PLATES
}

\author{
A. Kadir YAVUZ and Temel KOTIL \\ Istanbul Technical University \\ Aeronautics and Astronautics Faculty \\ Maslak 80626 İstanbul
}

\begin{abstract}
In this study, free vibration analysis of rotating adhesively bonded specially orthotropic plates is investigated. A first-order shear-deformation plate theory including geometric nonlinearities in the form of the von Karman strains and the effects of rotatory inertia and Coriolis is used to predict the free vibration frequencies and mode shapes of the system under consideration. In the analytical model, the thin interlaminar adhesive layer is taken into account in the form of mechanical springs. The governing non-linear equations of motion and the appropriate boundary conditions are derived by means of the variational method in conjunction with Hamilton's Principle.
\end{abstract}

\section{INTRODUCTION}

The dynamic characteristic of rotating plates find applications in flight and space vehicle structures and engine components such as aircraft propellers, wind turbine blades, and arm-like components of spacecrafts. The natural frequencies of rotating plates are governed by some additional parameters compared to those of non-rotating ones rotational speed and hub radius.

This subject has attracted the attention of numerous researches and a large number of studies are performed about the vibration analysis of rotating beams and plates in the engineering literature. Two recent literature surveys on this subject may be mentioned. Hodges [1] and Leissa [2] reviewed the papers on helicopter rotor blades and turbomachinery blades, respectively.

Since low-aspect ratio blades behave like plates rather than beams, a plate model will give better results for propellers. Bhumbla et al. [3], Dokainish and Rawtani [4], Ramamurti and Kielb [5], Wang et al. [6] and Show et al. [7] have studied vibrations of rotating plates.

Based on the author's knowledge, formulations of free vibrations of rotating multi-layer plates have never been reported in engineering literature. In this study, plates are adhesively bonded. Erdogan and Ratwani [8] and Yuceoglu and Updike [9] have studied on adhesively bonded structures and the modelling of adhesive bonding. Toghi has analysed the non-rotating multi-layer adhesively bonded plates [10] and Yavuz [11] and 
Yuceoglu et al. [12-14] have found the natural vibration characteristics of rotating multilayer adhesively bonded Euler and Timoshenko beams.

Therefore, the main objective of this study is to derive the governing equations of motion of adhesively bonded multi-layer rotating specially orthotropic plates while considering the effects of the transverse shear deformation, rotatory inertia and Coriolis by means of the variational method in conjunction with the Hamilton's Principle. A first order, nonlinear theory of plates with transverse shear deformation has been used for the analysis.

\section{THEORY}

According to the first-order shear-deformation theory, the in-plane displacements are functions of first-order powers of the thickness coordinate $z$ and the transverse shear strains, hence, the transverse shear stresses are constant through the thickness. Thus, the displacement field are expressed as [3]

$$
\left\{\begin{array}{l}
\bar{u}(x, y, z, t)=u(x, y, t)+z \psi_{x}(x, y, t) \\
\bar{v}(x, y, z, t)=v(x, y, t)+z \psi_{y}(x, y, t) \\
\bar{w}(x, y, z, t)=w(x, y, t)
\end{array}\right.
$$

where $\mathrm{u}, \mathrm{v}$ and $\mathrm{w}$ are the displacements at the midplane and $\psi_{x}$ and $\psi_{y}$ are negative of rotations about $y$ and $x$ axes, respectively.

Using the von Kármán theory of plates and Eq. (1) give the following non-zero strains become

$$
\left\{\begin{array}{l}
\varepsilon_{x}=\frac{\partial \bar{u}}{\partial x}+\frac{1}{2}\left(\frac{\partial w}{\partial x}\right)^{2}=\frac{\partial u}{\partial x}+\frac{1}{2}\left(\frac{\partial w}{\partial x}\right)^{2}+z \frac{\partial \psi_{x}}{\partial x} \\
\varepsilon_{y}=\frac{\partial \bar{v}}{\partial y}+\frac{1}{2}\left(\frac{\partial w}{\partial y}\right)^{2}=\frac{\partial v}{\partial y}+\frac{1}{2}\left(\frac{\partial w}{\partial y}\right)^{2}+z \frac{\partial \psi_{y}}{\partial y} \\
\gamma_{x y}=\frac{\partial u}{\partial y}+\frac{\partial w}{\partial x}+\frac{\partial w}{\partial x} \frac{\partial w}{\partial y}=\frac{\partial u}{\partial y}+\frac{\partial v}{\partial x}+z\left(\frac{\partial \psi_{x}}{\partial y}+\frac{\partial \psi_{y}}{\partial x}\right)+\frac{\partial w}{\partial x} \frac{\partial w}{\partial y} \\
\gamma_{y z}=\frac{\partial w}{\partial z}+\frac{\partial w}{\partial y}+\frac{\partial w}{\partial y} \frac{\partial w}{\partial z}=\psi_{y}+\frac{\partial w}{\partial y} \\
\gamma_{x z}=\frac{\partial u}{\partial z}+\frac{\partial w}{\partial x}+\frac{\partial w}{\partial x} \frac{\partial w}{\partial z}=\psi_{x}+\frac{\partial w}{\partial x}
\end{array}\right.
$$

and non-zero stresses for specially orthotropic plates are given by [10]

$$
\left\{\begin{array}{lr}
\sigma_{x}=B_{11} \varepsilon_{x}+B_{12} \varepsilon_{y}, & \sigma_{y}=B_{21} \varepsilon_{x}+B_{22} \varepsilon_{y} \\
\tau_{y z}=\kappa B_{44} \gamma_{y z}, & \tau_{x z}=\kappa B_{55} \gamma_{x z}, \quad \tau_{x y}=B_{66} \gamma_{x y}
\end{array}\right\}
$$


where

$$
\left\{\begin{array}{l}
B_{11}=\frac{E_{1}}{1-v_{12} v_{21}}, \quad B_{22}=\frac{E_{2}}{1-v_{21} v_{12}}, \quad B_{44}=G_{23} \\
B_{55}=G_{13}, \quad B_{66}=G_{12}, \quad B_{12}=B_{21}=v_{21} B_{11}=v_{12} B_{22}
\end{array}\right\}
$$

here $E$ and $G$ are moduli of elasticity and rigidity, respectively, $v$ is Poisson's ratio and subscripts denote the directions in which they are meant, and $\kappa$. is the shear correction factor.

In the adhesive layer, from Fig. 1 non-zero strains and stresses are written by substituting Eq. (1) as follows [10]

$$
\left\{\begin{array}{ll}
\varepsilon_{z}^{a}=\frac{\left.\bar{w}_{1}\right|_{z=-h_{1} / 2}-\left.\bar{w}_{2}\right|_{z=h_{2} / 2}}{h^{a}}=-\frac{w^{(2)}-w^{(1)}}{h^{a}}, & \sigma_{z}^{a}=E^{a} \varepsilon_{z}^{a} \\
\gamma_{x z}^{a}=\frac{\left.\bar{u}_{1}\right|_{z=-h_{1} / 2}-\left.\bar{u}_{2}\right|_{z=h_{2} / 2}}{h^{a}}=-\frac{1}{h^{a}}\left[u^{(2)}-u^{(1)}-\left(\frac{h_{1}}{2} \psi_{x}^{(1)}+\frac{h_{2}}{2} \psi_{x}^{(2)}\right)\right], & \tau_{x z}^{a}=G^{a} \gamma_{x z}^{a} \\
\gamma_{y z}^{a}=\frac{\left.\bar{v}_{1}\right|_{z=-h_{1} / 2}-\left.\bar{v}_{2}\right|_{z=h_{2} / 2}}{h^{a}}=-\frac{1}{h^{a}}\left[v^{(2)}-v^{(1)}-\left(\frac{h_{1}}{2} \psi_{y}^{(1)}+\frac{h_{2}}{2} \psi_{y}^{(2)}\right)\right], & \tau_{y z}^{a}=G^{a} \gamma_{y z}^{a}
\end{array}\right\}
$$

where the superscript $a$ indicates the adhesive layer, $h$ defines thickness of layers.

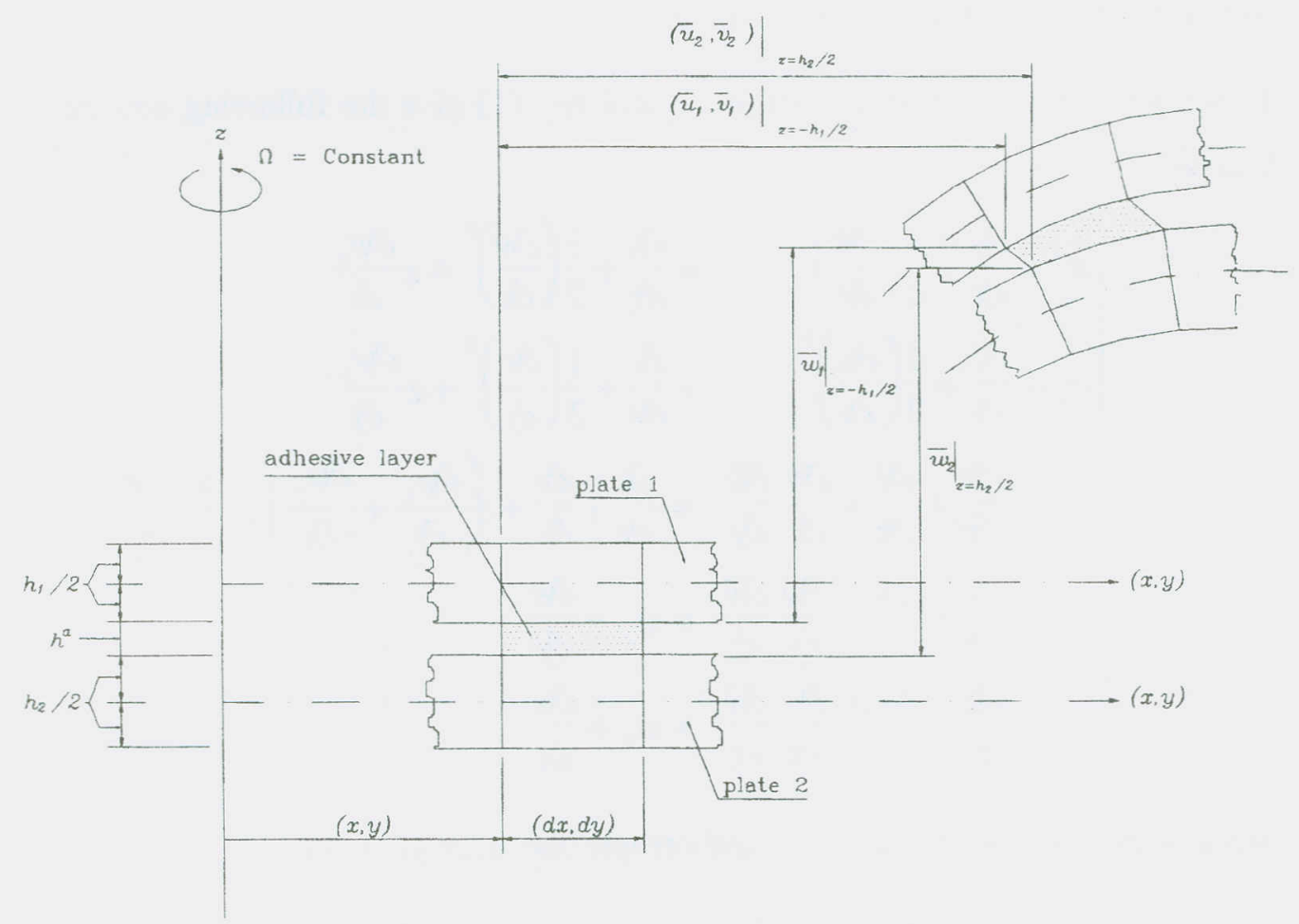

Fig. 1. Deformations of plates and interlaminar adhesive layer. 


\section{ENERGY EXPRESSIONS}

System under consideration has two plate layers and an adhesive layer which may be modeled as mechanical springs (Fig. 2). The strain energy expression for two plate layers are given by [3]

$$
U^{p}=\frac{1}{2} \sum_{i=1}^{2} \iint_{0}^{q} \int_{0}^{h} \int_{-h / 2}^{h / 2}\left(\sigma_{x}^{(i)} \varepsilon_{x}^{(i)}+\sigma_{y}^{(i)} \varepsilon_{y}^{(i)}+\tau_{x y}^{(i)} \gamma_{x y}^{(i)}+\tau_{y z}^{(i)} \gamma_{y z}^{(i)}+\tau_{x z}^{(i)} \gamma_{x z}^{(i)}\right) d x d y d z
$$

where $a$ and $b$ are dimensions of plates and the strain energy of the adhesive layer is

$$
{ }^{a}=\frac{1}{2} \iint_{0}^{a} \int_{0-h / 2}^{h / a}\left(\sigma_{z}^{a} \varepsilon_{z}^{a}+\tau_{y z}^{a} \gamma_{y z}^{a}+\tau_{x z}^{a} \gamma_{x z}^{a}\right) d x d y d z
$$

Then, the total strain energy of system becomes

$$
U=U^{p}+U^{a}
$$

Since the adhesive layer is assumed as very thin in comparison with the plate layers, its contribution to the kinetic energy of the system may be neglected. Then the total kinetic energy of the system may be found from

$$
T=\frac{1}{2} \sum_{i=1}^{2} \iint_{0}^{a} \int_{0}^{h / 2} \int_{-h / 2}^{h} \rho_{i}\left(\frac{\partial \vec{r}^{(i)}}{\partial t}\right)^{2} d x d y d z
$$

where $\rho$ is mass density of the plates and $\vec{r}$ is the position vector which is given by

$$
\vec{r}=(x+\bar{u}) \vec{i}+(y+\bar{v}) \vec{j}+(z+\bar{w}) \vec{k}
$$

where $\vec{i}, \vec{j}, \vec{k}$ are unit vectors in $x, y, z$ directions, respectively.

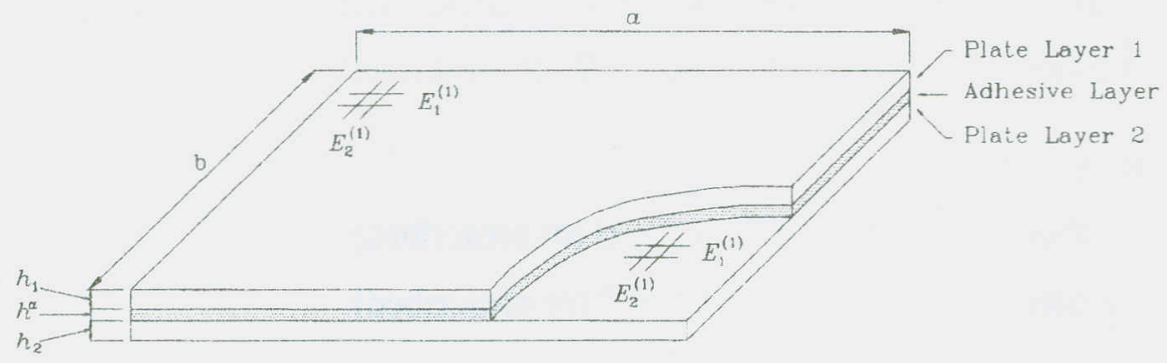

Fig. 2. General system configuration.

\section{DERIVATION WITH HAMILTON'S PRINCIPLE}

Governing equations for rotating specially orthotropic multi-layer adhesively bonded (or connected by mechanical springs) plates are derived via Hamilton's principle where 


$$
\delta \int_{t_{1}}^{t_{1}}(U-T) d t=0
$$

Substituting Eqs. (7) and (8) into Eq. (9) considering Eqs. (1-6) and making some manipulations involving variational calculus give the following governing equations $=1,2)$ :

$$
\begin{aligned}
& \rho_{i}\left[-\frac{\partial^{2} u^{(i)}}{\partial t^{2}}+2 \Omega \frac{\partial{ }^{(i)}}{\partial}+\Omega^{2}\left(x+u^{(i)}\right)\right]-(-1)^{i} \frac{f_{x}}{h_{i}}+\frac{\partial n_{x}^{(i)}}{\partial x}+\frac{\partial n_{x y}^{(i)}}{\partial y}=0 \\
& \rho_{i}\left[-\frac{\partial^{2} v^{(i)}}{\partial^{2}}-2 \Omega \frac{\partial u^{(i)}}{\partial t}+\Omega^{2}\left(y+v^{(i)}\right)\right]-(-1)^{i} \frac{f_{y}}{h_{i}}+\frac{\partial n_{y}^{(i)}}{\partial y}+\frac{\partial n_{x y}^{(i)}}{\partial x}=0 \\
& \rho_{i}\left(-\frac{\partial^{2} w^{(i)}}{\partial t^{2}}\right)-(-1)^{i} \frac{f_{z}}{h_{i}}+\frac{\partial}{\partial x}\left(n_{x}^{(i)} \frac{\partial w^{(i)}}{\partial x}+n_{x y}^{(i)} \frac{\partial w^{(i)}}{\partial y}+q_{x}^{(i)}\right)+\frac{\partial}{\partial y}\left(n_{y}^{(i)} \frac{\partial w^{(i)}}{\partial y}+n_{x y}^{(i)} \frac{\partial w^{(i)}}{\partial x}+q_{y}^{(i)}\right)=0 \\
& \frac{h_{i}^{2} \rho_{i}}{12}\left(-\frac{\partial^{2} \psi_{x}^{(i)}}{\partial t^{2}}+2 \Omega \frac{\partial \psi_{y}^{(i)}}{\partial t}+\Omega^{2} \psi_{x}^{(i)}\right)+\frac{f_{x}}{2}-q_{x}^{(i)}+\frac{\partial m_{x}^{(i)}}{\partial x}+\frac{\partial m_{x y}^{(i)}}{\partial y}=0 \\
& \frac{h_{i}^{2} \rho_{i}}{12}\left(-\frac{\partial^{2} \psi_{y}^{(i)}}{\partial t^{2}}-2 \Omega \frac{\partial \psi_{x}^{(i)}}{\partial t}+\Omega^{2} \psi_{y}^{(i)}\right)+\frac{f_{y}}{2}-q_{y}^{(i)}+\frac{\partial m_{y}^{(i)}}{\partial y}+\frac{\partial m_{x y}^{(i)}}{\partial x}=0
\end{aligned}
$$

and appropriate boundary conditions $(i=1,2)$ :

$$
\begin{aligned}
& \text { at } x=0, a \\
& \left\{\begin{array}{llll}
\text { either } & n_{x}^{(i)}=0 & \text { or } & u^{(i)}=0 \text { (or prescribed) } \\
\text { either } & n_{x y}^{(i)}=0 & \text { or } & v^{(i)}=0 \text { (or prescribed) } \\
\text { either } & n_{x}^{(i)} \frac{\partial w^{(i)}}{\partial x}+n_{x y}^{(i)} & \frac{\partial w^{(i)}}{\partial y}+q_{x}^{(i)}=0 & \text { or } \quad w^{(i)}=0 \text { (or prescribed) } \\
\text { either } & m_{x}^{(i)}=0 & \text { or } & \psi_{x}^{(i)}=0 \text { (or prescribed) } \\
\text { either } & m_{x y}^{(i)}=0 & \text { or } & \psi_{y}^{(i)}=0 \text { (or prescribed) }
\end{array}\right.
\end{aligned}
$$

at $\mathrm{y}=0, b$

$$
\left\{\begin{array}{lll}
\text { either } & n_{x y}^{(i)}=0 \quad \text { or } \quad u^{(i)}=0 \text { (or prescribed) } \\
\text { either } & n_{y}^{(i)}=0 \quad \text { or } \quad v^{(i)}=0 \text { (or prescribed) } \\
\text { either } & n_{y}^{(i)} \frac{\partial w^{(i)}}{\partial y}+n_{x y}^{(i)} \frac{\partial w^{(i)}}{\partial x}+q_{y}^{(i)}=0 \quad \text { or } \quad w^{(i)}=0 \text { (or prescribed) } \\
\text { either } & m_{x y}^{(i)}=0 \text { or } \quad \psi_{x}^{(i)}=0 \text { (or prescribed) } \\
\text { either } & m_{y}^{(i)}=0 \text { or } \quad \psi_{y}^{(i)}=0 \text { (or prescribed) }
\end{array}\right.
$$

where 


$$
\left\{\begin{array}{l}
f_{x}=k^{s}\left[u^{(2)}-u^{(1)}-\left(\frac{h_{1}}{2} \psi_{x}^{(1)}+\frac{h_{2}}{2} \psi_{x}^{(2)}\right)\right], f_{y}=k^{s}\left[v^{(2)}-v^{(1)}-\left(\frac{h_{1}}{2} \psi_{y}^{(1)}+\frac{h_{2}}{2} \psi_{y}^{(2)}\right)\right] \\
f_{z}=k^{n}\left(w^{(2)}-w^{(1)}\right), \quad n_{x}=B_{11}\left[\frac{\partial u}{\partial x}+\frac{1}{2}\left(\frac{\partial w}{\partial x}\right)^{2}\right]+B_{12}\left[\frac{\partial}{\partial y}+\frac{1}{2}\left(\frac{\partial w}{\partial y}\right)^{2}\right] \\
n_{y}=B_{21}\left[\frac{\partial u}{\partial x}+\frac{1}{2}\left(\frac{\partial w}{\partial x}\right)^{2}\right]+B_{22}\left[\frac{\partial}{\partial y}+\frac{1}{2}\left(\frac{\partial w}{\partial y}\right)^{2}\right], \quad n_{x y}=B_{66}\left(\frac{\partial u}{\partial y}+\frac{\partial v}{\partial x}+\frac{\partial w}{\partial x} \frac{\partial w}{\partial y}\right) \\
m_{x}=\frac{h^{2}}{12}\left(B_{11} \frac{\partial \psi_{x}}{\partial x}+B_{12} \frac{\partial \psi_{y}}{\partial y}\right), \quad m_{y}=\frac{h^{2}}{12}\left(B_{21} \frac{\partial \psi_{x}}{\partial x}+B_{22} \frac{\partial \psi_{y}}{\partial y}\right) \\
m_{x y}=\frac{h^{2}}{12} B_{65}\left(\frac{\partial \psi_{x}}{\partial y}+\frac{\partial \psi_{y}}{\partial x}\right), \quad q_{x}=\kappa B_{55}\left(\psi_{x}+\frac{\partial w}{\partial x}\right), \quad q_{y}=\kappa B_{44}\left(\psi_{y}+\frac{\partial w}{\partial y}\right)
\end{array}\right\}
$$

where $k^{n}$ and $k^{s}$ are normal and shear spring constants per unit volume which convert the adhesive layer constants to an equivalent mechanical spring constants in the modeling of problem (Fig. 3) and they are defined by

$$
k^{n}=\frac{E^{a}}{\left(h^{a}\right)^{2}}, \quad k^{s}=\frac{G^{a}}{\left(h^{a}\right)^{2}}
$$

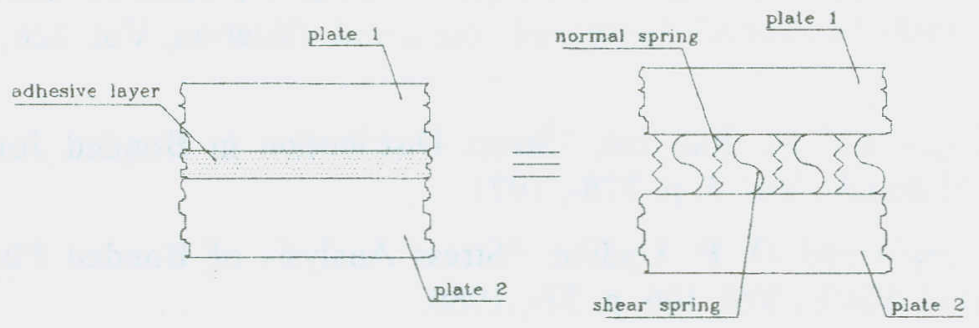

Fig. 3. The mathematical model of interlaminar adhesive layer by mechanical springs.

Eq. (10) consists of 10 coupled non-linear equations. After applying a suitable linearization peocess and assuming harmonic motion, these equations may be converted to a generalized eigenvalue problem and solved by means of some numerical methods such as finite difference, finite element, Rayleigh-Ritz, Galerkin-Vlasov's methods, etc. .

\section{CONCLUSIONS}

The governing non-linear equations of motion and appropriate boundary conditions of adhesively bonded multi-layer rotating specially orthotropic plates to be solved for finding the free vibration characteristics of system are derived while considering the effects of the transverse shear deformation, rotatory inertia and Coriolis by means of the variational method in conjunction with the Hamilton's Principle. A first order, non-linear 
theory of plates with transverse shear deformation has been used for the analysis. Adhesive layer is modeled by mechanical springs.

As a further study, by using these general equations, a suitable linearization process may be applied and numerical solutions may be obtained to be performed parametric studies.

\section{REFERENCES}

[1] D. H. Hodges, "Review of Composite Rotor Blade Modelling", AIAA Journal, Vol. 28, No.3, p. 561-565, 1990.

[2] A. Leissa, "Vibrational Aspects of Rotating Turbomachinery Blades", Applied Mechanics Reviews, Vol. 34, No. 5, p. 629-635, 1981.

[3] R. Bhumbla, J. B. Kosmatka and J. N. Reddy, "Free Vibration Behavior of Spinning Shear Deformable Plates Composed of Composite Materials", AIAA Journal, Vol. 28, No. 11, pp. 1962-1970, November 1990.

[4] M. A. Dokainish and S. Rawtani, "Vibration Analysis of Rotating Cantilever Plates", Int. Journal for Numerical Methods in Engineering, Vol. 3, No. 2, pp. 233-248, 1971.

[5] V. Ramamurti and R. Kielb, "Natural Frequencies of Twisted Rotating Plates", Journal of Sound and Vibration, Vol. 97, No. 3, p. 429-449, 1984.

[6] J. T. Wang, D. Shaw and O. Mahrenholtz, "Vibration of Rotating Rectangular Plates", Journal of Sound and Vibration, Vol. 112, No. 3, p. 455-468, 1987.

[7] D. Shaw, K. Y. Shen and J. T. Wang, "Flexural Vibration of Rotating Rectangular Plates of variable Thickness", Journal of Sound and Vibration, Vol. 126, No. 3, p. 373$385,1988$.

[8] F. Erdogan and M. Ratwani, "Stress Distribution in Bonded Joints", Journal of Composite Materials, Vol. 5, p. 378-, 1971.

[9] U. Yuceoglu and D. P. Updike, "Stress Analysis of Bonded Plates and Joints", Proceedings of ASCE, Vol. 106, p. 37-, 1980.

[10] F. Toghi, "Free Bending Vibrations of Adhesively Bonded Orthotropic Plates", Yüksek Lisans Tezi, Orta Doğu Teknik Üniversitesi, 1992.

[11] A. Yavuz, "Free Vibration Analysis of Multi-layer Adhesively Bonded Rotating Beams", Yüksek Lisans Tezi, Orta Doğu Teknik Üniversitesi, 1992.

[12] U. Yuceoglu, O. Tekinalp and A. Yavuz, "Natural Vibrations and the Orthogonality Condition in Rotating Composite Multi-layer Beams and Blades", Proceedings of the 22nd Midwestern Mechanics Conference (at the University of Missouri-Rolla, Rolla, U. S. A.), pp. 308-309, Oct. 1991.

[13] U. Yuceoglu, O. Tekinalp and A. Yavuz, "Natural Vibration Characteristics of Rotating, Composite Multi-layer Beams and Blades", Proceedings of the Fifth International Conference on Recent Advances in Structural Dynamics, University of Southampton, Southampton, England, Vol. 1, pp. 504-514, 18-21 July 1994.

[14] U. Yüceoğlu, O. Tekinalp and A. Yavuz, "Free Vibrations of Rotating, Adhesively Bonded Beams", International Symposium on Materials for Noise and Vibration Control, International Mechanical Engineering Congress and Exposition, 1994 ASME Winter Annual Meeting, Chicago, IL, U. S. A., November 6-11, 1994. 